\title{
Les valeurs d'ouverture à l'altérité dans les manuels de français libanais : aspects d'un traitement paradoxal
}

\section{Amandine Denimal}

\section{(2) OpenEdition}

\section{Journals}

Édition électronique

URL : http://journals.openedition.org/trema/2658

DOI : 10.4000/trema.2658

ISSN : 2107-0997

Éditeur

Faculté d'Éducation de l'université de Montpellier

Édition imprimée

Date de publication : 1 décembre 2011

Pagination : $36-53$

ISSN : 1167-315X

Référence électronique

Amandine Denimal, « Les valeurs d'ouverture à l'altérité dans les manuels de français libanais aspects d'un traitement paradoxal », Tréma [En ligne], 35 - 36 | 2011, mis en ligne le 01 décembre 2013, consulté le 01 mai 2019. URL : http://journals.openedition.org/trema/2658 ; DOI : 10.4000/ trema.2658

Ce document a été généré automatiquement le 1 mai 2019.

Trema 


\title{
Les valeurs d'ouverture à l'altérité dans les manuels de français libanais : aspects d'un traitement paradoxal
}

\author{
Amandine Denimal
}

1 Entre étude des représentations et étude des idéologies, l'étude des valeurs peine encore à définir son propre objet. Pourtant, les orientations axiologiques des discours scolaires, en particulier ceux des manuels, ne cessent de susciter débats et réflexions. C'est qu'à travers leurs textes, leurs images et leurs approches pédagogiques, les manuels véhiculent des conceptions de et sur la société qui les élabore et les adopte. Situés au carrefour des politiques nationales et des apprentissages des élèves, ils sont de plus en plus fortement perçus comme des transmetteurs de visions du monde qui, loin d'être neutres, reflètent

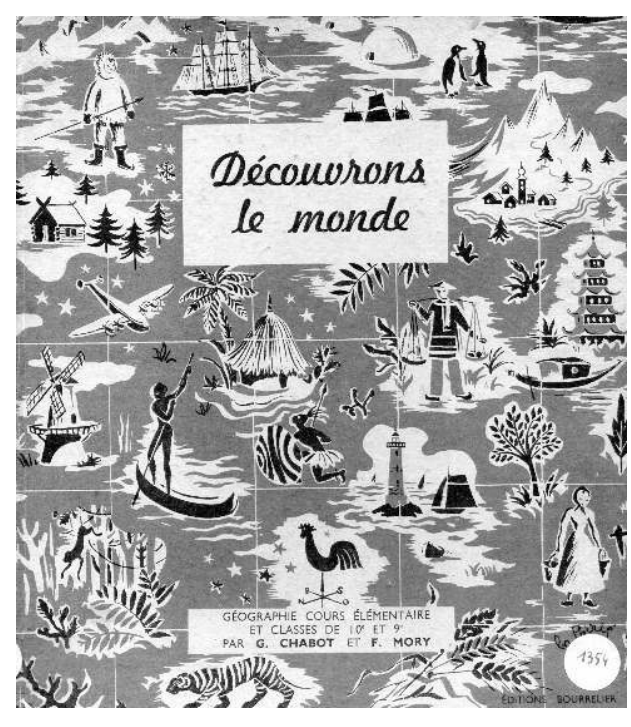
parfois davantage un projet national qu'une description objective de réalités humaines et sociales. Ce projet national contient toujours une préoccupation pour le sens moral des enseignements, qui s'énonce dans les manuels à travers des modèles de vivre ensemble ${ }^{1}$ qui répondent à l'exigence de socialisation citoyenne émanant de l'institution. Car les valeurs éducatives, qu'on trouve souvent explicitement énoncées dans les textes officiels, empruntent dans ces livres des voies plus secrètes et se décryptent dans ces modèles relationnels qui sont autant de discours tenus par l'école sur les relations sociales idéales entre les futurs citoyens, destinataires de ces discours. 
2 Au Liban, pays pluriconfessionnel ayant récemment traversé de longues années de guerre civile (1975-1990), les textes officiels de la réforme éducative entreprise au lendemain du conflit préconisent la formation d'un citoyen tolérant et respectueux des autres, à la fois attaché à son pays et ouvert sur le monde, tandis que les programmes de français laissent apparaitre un net intérêt pour les valeurs d'altérité.

La discipline "français " semble ainsi appelée à jouer un rôle particulier dans la transmission d'une vision du monde teintée de valeurs altruistes, tout en véhiculant des représentations positives du Liban. Ce souci de développer chez les élèves des attitudes positives à la fois en direction de la communauté nationale et des communautés étrangères n'a dans l'absolu rien de nouveau ni d'original, que ce soit dans les manuels de langue ou dans les textes censés régir leur élaboration. Cependant "les descriptions scolaires $\|^{3}$ sont tout autant tributaires des intentions institutionnelles que d'autres contraintes, plus difficiles à déceler, liées au contexte sociopolitique. Ainsi, si l'intention est de former à la connaissance et au respect de l'altérité, de quelle altérité s'agit-il dans des manuels conçus et utilisés dans un contexte précis? Selon quelles relations sociales se présente-t-elle? Comment représenter ces relations dans un pays pluriconfessionnel récemment sorti d'une guerre largement intercommunautaire? Comment éduquer au rapport à l'altérité dans un tel contexte et comment y encourager une citoyenneté unifiée? La particularité du contexte libanais en fait un terrain privilégié d'étude de l'image que cette société se donne d'elle-même à l'école.

Afin de cerner cette image, nous examinerons, «en amont » des manuels, les objectifs programmatiques des textes officiels quant aux valeurs, puis nous décrirons les modes de représentation du Liban et de la société libanaise dans un corpus de douze manuels de français ${ }^{4}$. Nous y adjoindrons les représentations des pays francophones (France incluse), en tant qu'altérités culturelles auxquelles le manuel d'enseignement du français doit - en principe - donner accès; et nous verrons en effet que ces représentations offrent un contrepoint particulièrement intéressant aux représentations du Liban, entre "altérité interne » (enjeu des relations libanaises) et " altérité externe » (horizon culturel étranger privilégié par le manuel)5. Nous essaierons de cette façon faire émerger la dimension axiologique de l'éducation à l'altérité et le rôle de l'enseignement du français dans cette éducation aux valeurs.

\section{Corpus et méthode d'exploitation}

3 Notre corpus comprend des manuels de français première langue ${ }^{6}$, de la quatrième année du cycle primaire à la dernière année du cycle complémentaire (collège) ${ }^{7}$. Ces manuels se divisent en deux collections: l'une destinée à l'enseignement public et l'autre à l'enseignement privé8. Les livres de l'école publique sont des manuels uniques édités par le CNRDP (Centre national de la recherche et du développement pédagogique), organe du ministère de l'Éducation chargé de l'élaboration des curricula et des manuels, et ils sont obligatoires dans toutes les écoles publiques. La commission d'agrément du CNRDP se charge également d'autoriser ou non la mise sur le marché des manuels élaborés en direction du privé, en vérifiant leur conformité avec les curricula et les objectifs éducatifs généraux du Ministère. Nous avons donc procédé à un choix concernant les manuels du privé et nous avons choisi ceux qui étaient les plus utilisés dans les écoles : les manuels de la collection «Didac » coédités par la Librairie ANTOINE et HACHETTE - EDICEF. La Librairie ANTOINE est la plus importante maison d'édition scolaire du Liban et elle est pour l'instant 
la seule maison à proposer une collection complète de manuels, du préscolaire à la terminale.

Tous ces livres ont été réalisés entre la fin des années 1990 et le début des années 2000 par des équipes d'auteurs libanais ${ }^{9}$ et à destination exclusive des apprenants du pays. Ce sont les premiers manuels réalisés après la réforme de 1997/98, et, après être passés par une réédition, ils sont encore en usage aujourd'hui.

Pour les étudier nous avons procédé à une analyse de contenu, en relevant les occurrences du thème du Liban et des Libanais-e-s dans les textes, les images et les activités pédagogiques. Nous avons fait de même avec les thèmes de la France, des Français-e-s, des pays francophones et de la francophonie. Pour chacune des occurrences, nous avons relevé le thème de l'unité didactique à l'intérieur de laquelle elle apparaissait, puis les aspects des pays ou des sociétés qui étaient traités. Nous avons également examiné la source des documents lorsqu'elle y figurait.

\section{Aspects de la politique éducative libanaise : les valeurs d'ouverture à l'altérité dans les objectifs généraux du CNRDP}

6 Les manuels libanais sont conçus pour tous les apprenants sans distinction de confession ni de milieu social. L'éducation, affichée comme priorité nationale, est considérée dans les textes officiels ${ }^{10}$ comme une "œuvre collective diversifiée", c'est-à-dire menée de concert par plusieurs instances ${ }^{11}$. L'enjeu de l'unité politique et de la diversité sociale est donc important. Celle-ci apparaît comme officiellement assumée et positivée, mais devant tout de même converger vers un projet commun : l'unité du Liban. L'altérité n'est donc pas seulement envisagée à l'extérieur des frontières, elle se trouve aussi à l'intérieur, entre dix-huit communautés reconnues officiellement par le régime confessionnel (l'appartenance confessionnelle recoupant, dans ce contexte, des critères à la fois religieux et ethniques). Les textes officiels placent parmi les dimensions intellectuelles et humaines de leurs objectifs « la conviction que le patrimoine spirituel du Liban, incarné dans les religions monothéistes, est un héritage précieux qu'il faut préserver et défendre comme un modèle de coexistence active et d'ouverture intellectuelle et spirituelle et en tant que l'antipode des systèmes et des doctrines fondées sur la discrimination raciale et le fanatisme religieux ». Diversité doit donc rimer avec richesse et ouverture.

7 C'est ainsi que le contexte d'après-guerre a impulsé, dans les années 1990, une importante réforme éducative dont les enjeux sont autant civiques que pédagogiques et dont un des objectifs était d'élaborer des manuels adaptés à un contexte spécifiquement libanais. La formation citoyenne de ce projet met en avant, à travers toutes les disciplines scolaires, des valeurs d'ouverture, de connaissance de l'Autre et d'acceptation des différences afin d'enrayer le repli des communautés sur elles-mêmes généré par la guerre civile.

Les objectifs généraux du CNRDP mettent ainsi en avant «l'unité nationale » et le « pacte de coexistence nationale ", qui passent par le respect d'autrui et des valeurs qui y sont attachées. Plusieurs passages du texte mentionnent l'importance des relations sociales et l'intérêt de la prise en compte de l'altérité : il s'agit de «donner [à l'apprenant] une attitude appréciative à l'égard [...] d'autrui, que cet autre soit un individu, une collectivité ou une nation ». L'éducation à l'unité nationale suppose un "attachement à la culture nationale et à l'ouverture aux autres cultures, aux valeurs humaines et à la modernité, cet 
attachement constituant une participation active à ces cultures et une source de développement et d'enrichissement mutuels ». Unité nationale et ouverture à l'altérité se conjuguent même dans cet attachement à la « culture nationale » et dans la connaissance de l'histoire commune, l'apprenant idéal devant être «imprégné » de son histoire nationale collective, rejetant tout sectarisme étroit et aspirant à une société unifiée et ouverte à toute l'humanité ». On lit donc dans ces lignes le désir de s'appuyer sur une tradition libanaise d'ouverture à autrui : les valeurs prônées de liberté, de tolérance et de respect d'autrui le sont aussi bien en tant que "valeurs humaines » - ayant une dimension universelle - qu'en tant que valeurs locales relevant de la culture libanaise. C'est un véritable accord entre respect de la nation et respect des autres cultures qui est visé, l'ouverture à l'une permettant l'ouverture aux autres et vice versa. On notera le caractère très large des dénominations "toute l'humanité " et "autres cultures ", que l'on retrouve dans le paragraphe relatif à la maîtrise d'au moins une langue étrangère, "pour une meilleure ouverture aux cultures du monde, source d'enrichissement mutuel ». Le caractère généralisant, pluriel et indéfini de cette dernière citation est certes caractéristique des textes officiels ; néanmoins, il peut nous laisser penser que le manuel de langue étrangère ne sera pas centré sur une culture en particulier (la culture française par exemple, la France étant le pays francophone avec lequel le Liban a des liens privilégiés), mais pourra viser des ensembles culturels plus larges ou même l'universalité. L'on peut donc, à partir de ce qui précède, faire état de deux options prises par les concepteurs d'objectifs éducatifs : premièrement le discours scolaire sur le Liban doit présenter ce pays comme un modèle d'ouverture à autrui ; deuxièmement l'enseignement des langues, en faisant connaître des cultures étrangères, doit promouvoir des valeurs altruistes dont la vocation est de contribuer à l'amélioration des relations entre Libanais, en proposant un modèle relationnel distancié ayant des effets vertueux en matière d'ouverture à l'altérité.

Mentionnons encore un aspect important de la réforme de l'enseignement du français : l'organisation des contenus d'enseignement selon une catégorisation thématique, chaque manuel devant désormais être divisé en unités traitant chacune d'un thème socioculturel précis. Les buts de ce remaniement étaient au nombre de trois : il s'agissait premièrement de privilégier une entrée dans la langue par le sens et non plus par la forme, deuxièmement d'inciter l'élève à lire et à s'exprimer sur son environnement et sur des sujets qui le touchent en ancrant les savoirs scolaires dans des thèmes volontairement actuels, et troisièmement de mettre en avant la promotion de valeurs. Ces différents thèmes ont donc été choisis en fonction des centres d'intérêt présumés des apprenants et des valeurs positives qu'ils étaient à même de véhiculer. On y trouve des thématiques «jeunes» (le sport, la musique...) couplées à des préoccupations plus résolument citoyennes telles que la connaissance et le respect du patrimoine, la protection de l'environnement, la culture scientifique et l'intérêt pour le progrès sous toutes ses formes (avec l'usage raisonné des technologies et des médias), l'amour du beau ou celui du travail ${ }^{12}$. D'autres thèmes sont spécifiquement consacrés au Liban ou à «l'ailleurs " (avec les thèmes du voyage, de l'évasion, des découvertes), sans spécification de destination. Une nette préoccupation pour le sens social et moral des contenus pédagogiques apparaît donc dans cette démarche en amont des nouveaux manuels, option qui remplace l'approche traditionnelle d'enseignement de la langue fondée sur les aspects linguistiques formels, l'histoire littéraire et la fréquentation des grands auteurs. Ces thèmes à la vocation axiologique affirmée nous semblent particulièrement fédérateurs pour des 
élèves issus de milieux socioculturels différents, car ils permettent $a$ priori de valoriser ce qui est commun aux différents Libanais, quelle que soit leur confession : le territoire, le patrimoine archéologique et naturel, l'art et les savoir-faire, ou encore de faire état des préoccupations liées à la mondialisation et à l'évolution des sociétés. Il importe désormais de voir comment ces thèmes sont effectivement traités dans les manuels.

\section{L'ouverture à l'altérité dans les manuels de français : traduction des objectifs officiels au niveau pédagogique}

11 Comment parle-t-on de sa nation à l'apprenant libanais ? Le Liban apparaît-il comme un modèle relationnel d'ouverture? Et que montre-t-on de la ou des cultures cibles pour favoriser cette ouverture?

\section{1. Représentations du Liban}

12 Une première constatation nous met sur la voie d'un paradoxe : la quasi absence de représentation de la société libanaise contemporaine. Alors même que les manuels sont censés faire connaître le Liban à l'apprenant et le promouvoir comme modèle d'ouverture sur les autres, ils se distinguent par le silence qu'ils entretiennent sur le Liban d'aujourd'hui dans ses aspects humains et sociaux. Les seuls aspects culturels dont ils font état sont neutres, ou plus exactement neutralisés par distanciation dans le temps, réification patrimoniale ou encore dilution dans les évolutions de la modernité. Bien entendu, le pluralisme confessionnel et la diversité sociale qui lui est associée constituent un sujet trop sensible pour être abordé par les manuels, si bien que pour insuffler l'amour du pays dans cette situation tendue, les procédés d'occultation d'une représentation objective des relations sociales actuelles usent d'une double stratégie: d'une part l'omission de tout ce qui a trait aux problèmes communautaires qui minent la société libanaise, et d'autre part l'inflation de discours sur des thèmes en apparence dépolitisés mais susceptibles de resserrer les liens entre Libanais: le territoire et son climat, le patrimoine naturel, le patrimoine historique, la littérature orientaliste et le conte, l'artisanat et les traditions, la mondialisation.

Ainsi les manuels, et tout particulièrement ceux de l'enseignement public, recourent fréquemment à un genre de description qui vante la beauté des paysages libanais, non sans insister sur leur diversité : une rencontre harmonieuse de montagnes, de mer et de plaines qui fait de ce cadre une exception géographique dans la région. Ainsi l'unité didactique «Visages du Liban » du manuel du CNRDP de la Septième année comprend, sur six textes, un texte (assorti d'une carte géographique) décrivant les aspects généraux du pays façon «carte d'identité » et deux textes sur le climat du Liban ${ }^{13}$. La valorisation du pays est bien présente, mais le type de diversité mis en avant n'est pas social.

13 Le patrimoine naturel est lui aussi valorisé par la description des principaux sites libanais, notamment celui des Cèdres, emblème national et exception régionale, ou celui de la grotte de Jeita. C'est également l'occasion de sensibiliser l'apprenant à la protection de l'environnement; un environnement volontiers libanais, comme en témoignent les sections «Les fleurs du Liban sont sa parure, protégeons-les », «Les oiseaux du Liban », «Une forêt libanaise » ou «Les animaux du Liban » dans l'unité « Nature » du manuel de 
la Septième année ${ }^{14}$, ou encore le texte "Les cèdres du Liban » dans l'unité "Attention, nature fragile! » du manuel de la Cinquième année ${ }^{15}$. Cette tendance est plus forte dans les manuels du CNRDP que dans les manuels de la Librairie ANTOINE, pour lesquels on peut tout de même citer le texte «Le cèdre, un arbre millénaire » et une activité d'exposé oral sur trois sites naturels libanais dans l'unité « Soyez polis... avec la Terre et ses arbres, la mer et ses oiseaux" du manuel de quatrième année ${ }^{16}$, ainsi que le texte "La forêt d'Ehden » dans celui de la Cinquième année ${ }^{17}$, au sein de l'unité « La mémoire d'un pays ».

La description des sites historiques, notamment antiques, constitue un grand classique des manuels, que ce soit dans les textes, les images ou les activités pédagogiques; à ces hauts-lieux de l'histoire universelle reste attaché un rayonnement mondial important, et ils sont exploités comme autant de motifs de fierté nationale et d'attachement à une exception culturelle. Encore une fois, le phénomène est plus fréquent dans les manuels de l'enseignement public: citons, parmi d'autres exemples, l'unité «Explorations et découvertes ${ }^{18}$ ", qui offre trois textes (dont un de quatre pages) mettant en valeur les vestiges des civilisations anciennes qui se sont succédées sur le sol du Liban actuel, ou bien l'unité «Le patrimoine » du livre de Neuvième année, qui, en plus d'une section consacrée au patrimoine national (pp.70-71), consacre encore six pages aux sites libanais dans la section dédiée au patrimoine mondial. Les livres du CNRDP penchent donc résolument du côté du géographique, du factuel et de l'archéologique, ce qui neutralise fortement, voire réifie la vision du Liban ; cela peut s'expliquer par le fait que les écoles publiques sont non confessionnelles : les manuels s'adressent à un public forcément large, et les thèmes de société touchant de près l'actualité du pays n'en sont que plus difficiles à aborder. Il en résulte alors un curieux effet touristique ${ }^{19}$, le Liban apparaissant dans le manuel comme dans un dépliant publicitaire.

Les manuels de la Librairie ANTOINE, eux, livrent quelques photos de sites antiques, mais se concentrent davantage sur le patrimoine architectural plus récent (les maisons traditionnelles) encore présent dans les régions rurales, et dont l'origine étrangère de certains éléments est mentionnée. Un texte du manuel de Quatrième année, tiré lui aussi d'un guide touristique, est consacré au site de Deïr El-Kamar et s'achève sur ces mots : " Deïr El-Kamar est un des témoignages, en un lieu très circonscrit, de cette succession ininterrompue de civilisations et d'hommes, une des constantes de l'histoire libanaise » (p.84).

Dans cette phrase se trouve résumée une idée importante, que véhicule un grand nombre de ces textes à thématique historique: celle que le Liban s'est construit au gré de multiples influences, et ce depuis toujours. La question de l'origine, et donc de la légitimité d'une communauté par rapport à une autre, s'en retrouve pratiquement caduque, puisque les textes traitant du patrimoine archéologique permettent le plus souvent d'évoquer les différentes civilisations successivement établies sur la côte et les contreforts du Mont Liban, tout en valorisant - de façon nostalgique, peut-être - un passé prestigieux qui voyait l'influence des Phéniciens s'étendre au monde entier. On aurait finalement affaire à une forme de diversité culturelle, mais étalée dans le temps selon une logique de strates successives et relativement étanches ${ }^{20}$, semblables à celles mises au jour dans les chantiers de fouilles; une forme de représentation de la société libanaise actuelle? On y retrouve en tout cas l'enjeu de la connaissance de l'histoire nationale stipulée dans les textes officiels, une histoire présentée comme une collection d'influences multiples et enrichissantes, autrement dit la promotion d'un modèle social et 
politique - la diversité sociale dans l'unité nationale - dont les corollaires sont les valeurs de respect et de tolérance des différences.

Dans les deux types de manuels, on trouve des extraits de récits de voyages d'écrivains français des XVIIIe et XIXe siècles tels que VOLNEY, LAMARTINE, NERVAL ou FLAUBERT. Ils y décrivent une contrée représentant pour eux « l'Orient », ailleurs lointain et fantasmé, et leur fascination pour l'exotisme pointe sous la minutie des descriptions. Cependant ce choix de l'orientalisme semble répondre à un double objectif : valoriser le Liban par ce regard fasciné porté sur lui, et décrire une société des siècles passés. Le cas d'un manuel reproduisant trois textes traitant de noces chrétiennes ou musulmanes (LAMARTINE pour un mariage orthodoxe, NERVAL pour deux mariages musulmans en Égypte ${ }^{21}$ ) est particulièrement intéressant: des coutumes religieuses et culturelles différenciées sont évoquées, mais l'éloignement temporel et le caractère relativement littéraire de la scène décrite adoucissent le propos social. Il apparaît ainsi que la représentation du Liban passe par une double distanciation: celle du temps par le recours nécessaire à des contextes différents du présent, et celle des narrateurs étrangers français à qui l'on semble attribuer plus d'objectivité (voire de légitimité) pour parler du Liban.

Le conte est lui aussi bien représenté dans plusieurs niveaux d'enseignement et dans les deux types de manuels. C'est l'occasion de réinvestir l'imaginaire des contes orientaux de la Perse au Maghreb, à côté de contes occidentaux. Une communauté de références culturelles s'y met en place, des espaces de partage s'y dessinent tandis que le légendaire met à distance le réel et exploite des problématiques universelles. Ce procédé permet d'évoquer des aspects moraux universels touchant les relations humaines, avec un ancrage culturel minimal et transnational.

C'est cette dernière caractéristique que l'univers du conte partage avec l'orientalisme littéraire : une sorte de fusion dans un "ancrage culturel oriental global», qui prend en compte les références de l'apprenant sans particulariser les appartenances et sans créer des modèles d'identités fermées. Les écrits des orientalistes aussi bien que les contes ignorent les frontières contemporaines, promènent librement le regard du Machreq au Maghreb et décrivent un monde qui parait à la fois divers et unifié. Cela représenterait-til, pour le projet libanais d'unification nationale, une sorte de monde idéal, un espace décloisonné ou réconcilié, où les échanges et les dialogues seraient possibles?

Si l'un des enjeux de la description scolaire est d'unifier l'espace, un autre pourrait consister à unifier le temps. Dans une vision reliant passé et présent, le monde coloré des souks $^{22}$ laisse percevoir un Liban éternel, où les traditions se perpétuent. L'artisanat libanais et les traditions (en particulier culinaires) constituent ainsi un important réservoir de représentations ${ }^{23}$. Le texte informatif de nature touristique domine certes dans ce registre, mais certains textes littéraires ne sont pas en reste pour planter le décor dans une campagne éternelle, proche de ses tradition ${ }^{24}$. Des traditions décrites, bien sûr, sans distinction de milieux sociaux.

19 Enfin, la description scolaire prend pour objet des processus sociaux très globaux, signes de l'évolution actuelle des sociétés sous l'effet de la mondialisation. L'on présente ainsi les centres d'intérêt des jeunes Libanais actuels, semblables à ceux de bien d'autres jeunes du monde occidental: usage des technologies, intérêt pour la science fiction, monde de l'information et des médias... Les manuels prennent le parti de décrire une «culture jeune » sans frontières. 
20 Même si l'on observe des tendances différentes entre les deux collections de manuels (ceux du CNRDP penchent plus pour un Liban géographique et archéologique, ceux de la Librairie Antoine pour un Liban historique plus récent), les types de représentation que nous avons décrits sont identiques, et permettent la mise à distance de la société libanaise contemporaine: l'ancien (de l'Antiquité au XIXe siècle) est très représenté, et le contemporain est généralement présenté comme une continuité de l'ancien et des valeurs de coexistence pacifique qui lui sont attribuées. Cela permet de donner une image homogène - pour ne pas dire harmonieuse - du pays et de son histoire, à moins qu'on ne préfère conférer au Liban une dimension plus universelle, que ce soit par le monde ahistorique des contes (avec leurs morales applicables à tous les contextes) ou par l'inscription dans des dynamiques sociales mondiales. La vision qui s'en dégage est tout à fait apaisée, et les ferments de division neutralisés car l'on évite de représenter des cultures de façon trop différenciée et particularisante ; sans doute considère-t-on qu'un discours trop culturaliste serait difficilement contrôlable au niveau de sa réception. Favoriser de bonnes dispositions envers l'altérité libanaise, mais sans la désigner comme telle, tel est au Liban le défi de la description scolaire. Il nous reste à voir si cela se vérifie au niveau des représentations des altérités francophones.

\section{2. Représentations de la France et des autres pays francophones}

21 Les représentations de la France manifestent les mêmes tendances à la non particularisation du culturel. Ainsi, contrairement à toute attente, les manuels du Liban, qui a eu et a toujours des relations étroites avec la France, n'en parlent finalement que très peu - du moins en tant que pays ou culture spécifique. Un seul manuel se livre à une description explicitement centrée sur la France ${ }^{25}$, d'un point de vue strictement touristique et patrimonial ; le Livre de français de Septième année consacre un texte à Louis PASTEUR (pp.156-157), dont une image apparaît dans Au Pays des Mots quatrième année aux côtés de photos de Marie Curie et de Louis Blériot (p.17). D'autres figures célèbres (associées aux exploits sportifs ou aux découvertes scientifiques) sont évoquées de loin en loin, la plupart du temps sans que leur appartenance nationale ne soit mentionnée ; c'est plutôt leur valeur de modèle édifiant qui semble cultivée. La culture française, au sens anthropologique de pratiques et représentations sociales des Français, ne constitue donc apparemment pas un ancrage à partir duquel va se déterminer une véritable perception de l'altérité. Car même si un nombre très important de documents textuels provient de la littérature ou de la presse française, ils ne sont pas présentés comme tels, ni comme pouvant faire l'objet d'une lecture culturelle particulière nécessitant une contextualisation sociohistorique. Ils sont simplement présentés comme allant d'euxmêmes, comme évidents pour de jeunes apprenants libanais ; l'altérité qu'ils représentent est donc prise en compte de façon paradoxale, dans des manuels censés ouvrir sur une perception et une compréhension de l'Autre.

Il en va de même pour les autres pays francophones: leur production littéraire et médiatique est mise à profit, mais le paratexte fait silence sur les pays d'origine des auteurs, et sur les éventuelles spécificités culturelles dont le lecteur pourrait avoir besoin. Il faut dire que ces auteurs et journalistes francophones sont souvent libanais, et que des écrivains mondialement connus tels qu'Andrée CHEDID, Amin MAALOUf, Nadia TUENI, Farjallah HAÏK, Georges SHÉHADÉ ou Michel CHIHA figurent en bonne place dans les manuels 
des deux collections ; les autres auteurs d'expression française viennent pour la plupart des pays du Maghreb, comme Mouloud FERAOUN ou Tahar BEN JELLOUN.

Ce type d'enseignement culturel rapprocherait les manuels libanais des manuels de français langue seconde ${ }^{26} \mathrm{~d}^{\prime}$ Afrique francophone par exemple, où la culture cible fait généralement l'objet d'une "prise en compte malaisée » (VERDELHAN-BOURGADE, 2006). Loin de désigner une altérité culturelle, ces manuels présentent un monde francophone évident et d'emblée accessible. On peut donc se demander quel rôle remplissent exactement ces livres en matière d'ouverture à l'altérité et à la différence, si l'altérité n'est pas désignée comme telle et si la différence est passée sous silence. Cette prise en compte paradoxale de l'altérité francophone répondrait-elle, tout comme celle de l'altérité libanaise, aux exigences idéologiques de la description scolaire: favoriser le consensus? Ne pas désigner de différences, mais seulement des espaces de partage fondés sur l'évidence? Préférer au relativisme culturel une universalisation des cultures et de leurs valeurs?

\section{Prolongements et contrepoints interprétatifs : entretiens avec des auteurs de manuels et des concepteurs de programmes scolaires}

Une telle configuration mérite que l'on s'intéresse au point de vue de ceux qui sont le plus à même de répondre du contenu des manuels : leurs auteurs. Pour nous aider à cerner la raison d'être des représentations que nous avons relevées, nous avons décidé de les interroger et avons pu, au cours du mois de mai 2010, réaliser à Beyrouth neuf entretiens d'auteurs (six du CNRDP et trois de la Librairie ANTOINE). Ces auteurs sont soit des enseignants de français du niveau secondaire ou universitaire, soit des coordinateurs de français ${ }^{27}$, et certains d'entre eux ont participé, dans les années 1990, au groupe de réflexion sur la réforme éducative. Leur appartenance confessionnelle s'est parfois exprimée lors des interactions sans que nous l'ayons explicitement sollicité, et nous a laissé supposer une forte dominante chrétienne. Nous leur avons demandé quelle image ils avaient souhaité donner des pays que nous étudions, la raison d'être des différentes représentations que nous avions pu relever, et s'il y avait des valeurs éducatives particulières qu'ils avaient souhaité faire passer. Bien entendu il importe de relativiser les réponses obtenues, au regard de trois constats: premièrement, il ne s'agit que de déclarations, faites à propos d'un travail réalisé plusieurs années auparavant, et l'on sait que les intentions d'un auteur sont une donnée à nuancer lorsqu'on étudie un produit fini. Deuxièmement les auteurs en question ont œuvré collectivement, et sous la triple houlette d'un ministère de tutelle (ou du moins d'un cahier des charges officiel), d'un éditeur (voire de deux) et d'un co-ordinateur de collection, ce qui conduit à relativiser fortement leur responsabilité vis-à-vis des contenus. Troisièmement, l'origine française de l'enquêtrice a certainement joué un rôle dans l'interaction et influencé les réponses obtenues chez des auteurs majoritairement chrétiens: une connivence autour du caractère identitaire de la langue française pour les chrétiens libanais s'est possiblement exprimée $e^{28}$.

L'importance des valeurs de respect de l'altérité et d'acceptation des différences a fréquemment été soulignée par ces informateurs, pour qui la capacité des citoyens à vivre ensemble dans une société multiconfessionnelle et multiculturelle constitue la condition 
de $"$ la survie du Liban $»^{29}$. Pour certains d'entre eux, le Liban et son histoire peuvent même représenter un modèle adéquat de coexistence dans la diversité, puisqu'avant la guerre les différentes confessions vivaient en bonne intelligence. Concernant les représentations du pays, la volonté de créer une vision apaisée est unanime : un Liban contemporain, livré aux guerres intestines, ne conviendrait pas à la construction d'une socialisation scolaire et sociale viable. Les phénomènes d'occultation que nous avons observés dans les manuels sont donc présentés comme des choix délibérés : les auteurs disent avoir craint que la représentation de différences entre les Libanais ne contribue à exacerber les identifications communautaires au détriment du projet d'unification nationale et de coexistence pacifique. Le désir de représenter une diversité libanaise était néanmoins présent : cinq auteurs se sont référés à la diversité géographique du pays, que nous avons évoquée précédemment. Leur motivation a fait clairement ressortir une recherche de remédiation aux replis régionaux qui ont accompagné les replis communautaires durant la guerre : la connaissance des différentes régions du pays est censée encourager les apprenants à ne pas s'enfermer mentalement sur leurs territoires communautaires respectifs; reste que l'on a affaire à une ouverture à l'Autre libanais qui prend de préférence pour objet des données géographiques et factuelles. Quant à la sélection de textes de la littérature orientaliste dans les manuels de la Librairie ANTOINE, elle résultait elle aussi d'un choix délibéré, effectué selon une perspective bien particulière : une représentation ethnographique des us et coutumes relevant de diverses communautés religieuses du pays.

Concernant les représentations du monde francophone, une réticence à « objectiver » les entités «France » et "culture française » est apparue dans les entretiens, les auteurs interrogés considérant que le français n'est pas vraiment une langue étrangère au Liban (ce en quoi l'on retrouve la composante chrétienne des représentations); la considérer comme telle reviendrait à poser une altérité, ce qui ne siérait pas au statut qu'elle a hérité de l'histoire ni à la perception qu'en ont, d'après eux, les Libanais. Mais n'aurait-on pas affaire ici à une perception construite, projetée sur les usagers des manuels, et centrée du point de vue communautaire? Une perception dans laquelle les vocations différenciées des disciplines scolaires entrent en jeu? Plusieurs informateurs ont en effet fait état d'une partition des valeurs éducatives selon les disciplines d'enseignement: la langue arabe et les matières enseignées en arabe seraient chargées d'assurer la transmission des valeurs du monde arabe, tandis que le français et les matières enseignées en français auraient pour tâche de véhiculer des valeurs " occidentales " ${ }^{30}$ associées à l'universalité. L'enseignement du français semblerait donc, pour ces acteurs du système éducatif, avoir pour enjeu idéologique la transmission de valeurs altruistes dites universelles (liberté, tolérance, respect de l'Autre et des différences) et d'un système de références universalisé, non centré sur le local. Un type d'ouverture au monde dont la vocation n'est pas de susciter l'intérêt pour un ailleurs culturel et social différencié, qui se passe de démarche interculturelle en un mot. C'est ce que pourrait encore corroborer le choix des textes de la littérature francophone du monde arabe, choisis pour que l'apprenant libanais y retrouve des références culturelles familières dans une autre langue que sa langue maternelle.

Ces entretiens d'auteurs nous ont permis de mieux percevoir, sous l'aspect consensuel des discours scolaires, des moyens indirects de représentation du rapport à l'Autre. Ils nous ont également confortée dans l'hypothèse d'une occultation du Liban contemporain et d'une tendance à gommer le particulier dans la description scolaire : il en résulte une 
tendance à l'universalisation des valeurs et des modèles sociopolitiques occidentaux, tendance qu'assument ces auteurs. Les manuels du lycée prolongent d'ailleurs la représentation d'une francophonie ancrée dans un propos universalisant, à travers deux unités didactiques consacrées l'une à la francophonie et l'autre à l'humanisme. Cette francophonie des valeurs universelles est celle prônée par les institutions internationales de la Francophonie, et les manuels du public comme du privé manifestent une inclusion du Liban au sein de cet ensemble.

Pour compléter cette étude, il importerait d'explorer les représentations des autres espaces et groupes sociaux présents dans les manuels: nous n'y avons étudié que partiellement l'éducation à l'altérité, qui de surcroît ne peut se satisfaire d'une simple analyse des contenus thématiques; une analyse des discours de ces manuels est incontournable, de même qu'une étude de la réception de ces discours chez les apprenants permettrait sans doute d'en mesurer l'impact. Nous avons simplement cherché à donner un aperçu de la relation entre représentations culturelles et enjeux sociétaux au Liban, et à aborder la discipline « français » du point de vue de cette relation.

\section{Diversité sociale et unification nationale}

Le manuel scolaire est un discours traversé d'enjeux sociopolitiques et idéologiques. Éviter les sujets qui divisent les citoyens, fédérer les consciences grâce à l'école, présenter des modèles de socialisation à l'intérieur comme à l'extérieur des frontières tout en conservant une neutralité de mise vis-à-vis des problèmes réels de coexistence nationale, tels sont à l'école libanaise les enjeux de la représentation du monde social. Des paradoxes en émergent nécessairement: faire connaître et aimer le Liban, mais sans parler des aspects les plus brûlants de son actualité; le présenter comme modèle d'ouverture aux autres, mais sans représenter une diversité sociale susceptible de contredire cette ambition; donner une vision positive des différences entre les hommes, mais sans mentionner celles qui existent entre les Libanais d'aujourd'hui; s'ouvrir aux cultures étrangères, mais sans considérer le français comme langue étrangère; tels sont les aspects d'un paradoxe éducatif qui mène naturellement à l'énonciation de valeurs universelles plutôt qu'à une démarche interculturelle, selon une logique de l'exemple, du modèle à suivre pour des apprenants qui doivent réapprendre à aimer leur pays.

L'on pourrait taxer le tout d'une trop grande abstraction, assortie d'une neutralisation ou d'une occultation de la réalité, voire d'une distorsion de cette réalité au bénéfice de la vision d'une communauté. Mais éduquer à une citoyenneté unitaire après un conflit est loin d'être chose aisée, et ces manuels s'efforcent malgré tout de ne pas mettre en péril une fragile coexistence. Les partis pris des acteurs éducatifs manifestent une profonde et nécessaire entreprise d'apaisement, et choisissent, en contrepoint d'une difficile prise en compte du local et du présent, une forme de neutralité couplée à une universalisation des valeurs dont s'est doté le projet national. Sans omettre de laisser affleurer quelques espaces ténus d'échange et de partage, que ce soit au travers des influences culturelles diverses relevées dans l'architecture et dans le mode de vie, ou sous la plume des écrivains francophones du monde arabe. 


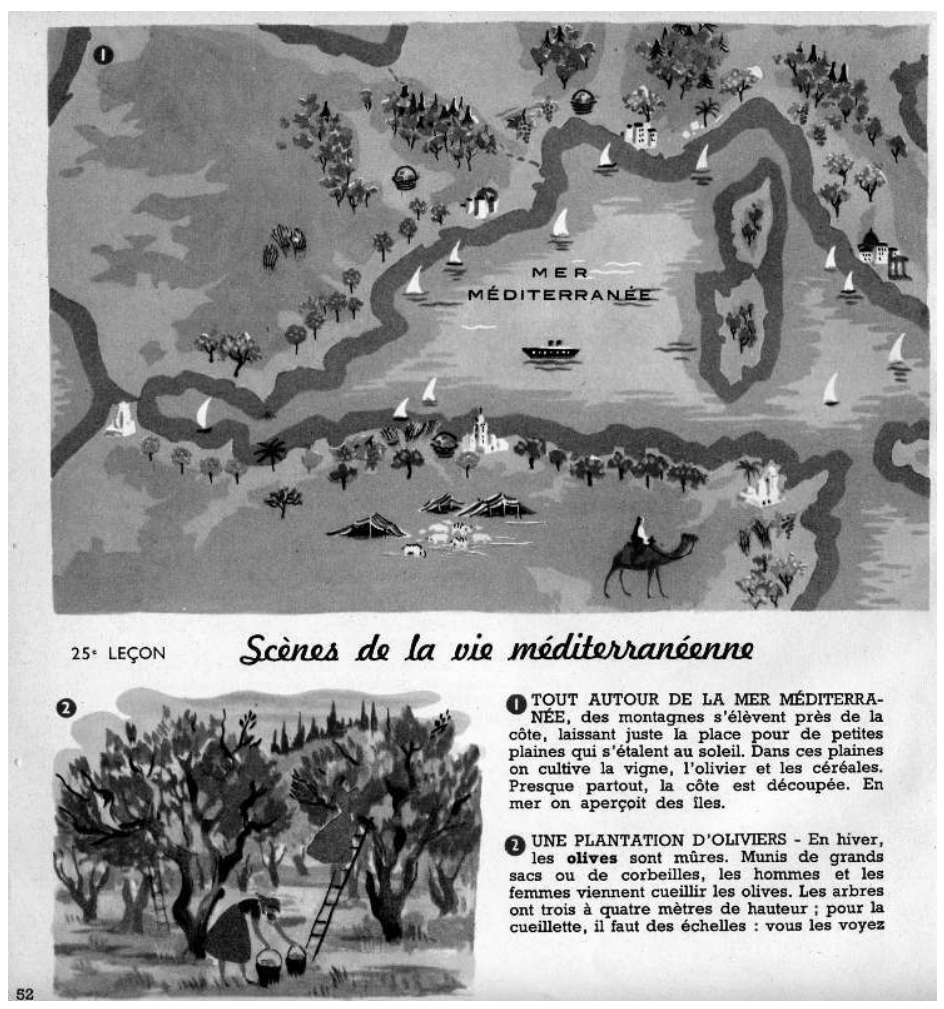

Découvrons le monde, G. CHABOT et F. MORY. Paris : Editions BOURRELIER, Imprimerie G. Lang. Lang, 1953, 80 p., p. 52 (Illustration H. POIRIÉ) - Source : CEDRHE.

\section{BIBLIOGRAPHIE}

AZAR F., Construction identitaire et appartenance confessionnelle au Liban, approche pluridisciplinaire. L'Harmattan. Collection Comprendre le Moyen-Orient, 1999 DEMOUGIN P., coord. Manuel et médiation. Discours didactiques $n^{\circ} 2$. Montpellier : Presses universitaires de la Méditerranée, 1998

HAFEZ S.-A., Statuts, emplois, fonctions, rôles et représentations du français au Liban. Paris : L'HARMATTAN, 2006

MAINGUENEAU D., Les livres d'école de la République : discours et idéologie. Paris : Le Sycomore, 1979

SINCLAIR M., Planifier l'éducation en situation d'urgence et de reconstruction. Paris : UNESCO, Bureau international de planification de l'éducation, 2003

Reshaping the future, education and post-conflict reconstruction. Washington : Rapport de la banque mondiale, 2005

VERDELHAN-BOURGADE M., L'interculturalité en français langue seconde, une prise en compte malaisée. In CONDEI, C., DUFAYS, J.-L. et LEBRUN, M. (éds), L'interculturel en francophonie. Représentations des apprenants et discours des manuels. Belgique : EME. Collection Proximitésdidactique, 2006

VERDELHAN-BOURGADE M., Les manuels scolaires, miroirs de la nation? Paris : L'Harmattan. Collection Langue et parole, 2007

ZARATE G., Représentations de l'étranger et didactique des langues, Paris : Didier-Crédif, 1993 


\section{Sitographie}

Site officiel du Centre National de la Recherche et du développement pédagogique : http:// www.crdp.org/CRDP/French/curriculum/curriculum.asp

\section{Corpus de manuels}

SARDOUK GHANDOUR N., AWAD, ITANI H., MOGHRABI H., SOULEIMAN E., Dire, lire, éducation de base $5 e$ année, Liban : CRPD. Le livre scolaire national, 1998

SARDOUK N., AWAD Z., AOUn BOUlos D., ITANI H., SOULEIMAN E., Dire, lire, éducation de base 5e année, Liban : CRPD. Le livre scolaire national, 1999

hanNa MaAlouf L., ATTAR ALlieh F., KHOURy ABi CHACRA M., AL RIFAÏ B., Dire, lire, éducation de base $6 e$ année, Liban : CRPD. Le livre scolaire national, 2000

AOUN R., ASSI I., PILLET-ABOU MEHRI A., HARIZ JABBOUR M., Livre de français, éducation de base 7e année (2e édition), Liban : CRPD. Le livre scolaire national, 1996

AOUN R., ASSI I., PILLET-ABOU MEHRI A., HARIZ JABBOUR M., Livre de français, éducation de base 8e année (2e édition), Liban : CRPD. Le livre scolaire national, 1997

AOUN R., ASSI, I., PILLET-ABOU MEHRI, A., HARIZ JABBOUR M., BEJJANI M., Livre de français, éducation de base 9e année, Liban : CRPD. Le livre scolaire National, 2000

ABOU GHANNAM A., BAGHDADI ADRA B., ATTIEH M., CHIKHANi R., FAWAZ A., HADDAD G., HOYeK S., MHANNA G., NACOUZ L., ROUPHAËL G., Au pays des mots, éducation de base 4e année, Liban : Librairie Antoine/ Hachette-Edicef. Collection Didac, 2003

ABOU GHANNAM A. et al., Au pays des mots, éducation de base 5e année (2e édition), Liban : Librairie Antoine/ Hachette-Edicef. Collection DIDAC, 2006

ABOU GHANNAM A. et al., Au pays des mots, éducation de base 5e année (2e édition), Liban : Librairie Antoine/ Hachette-Edicef. Collection DIDAC, 2007

ABOU GHANNAM A. et al., À la croisée des textes, éducation de base 7e année (2e édition), Liban : Librairie Antoine/ Hachette-Edicef. Collection DIDAC, 2005

ABOU GHANNAM A. et al., À la croisée des textes, éducation de base 8e année (2e édition), Liban : Librairie Antoine/ Hachette-Edicef. Collection DIDAC, 2005

ABOU GHANNAM A. et al., À la croisée des textes, éducation de base 9e année (2e édition), Liban : Librairie Antoine/ Hachette-Edicef. Collection DIDAC, 2008

\section{NOTES}

1. Pour Geneviève ZARATE, « les outils d'enseignement des langues vivantes présentent un intérêt sociologique particulier : ils mettent à plat la relation à l'étranger qu'une société donnée veut offrir en modèle à ceux qu'elle éduque » (1993: 11).

2. Elle est historiquement privilégiée au Liban, où elle représente généralement un nombre d'heures d'enseignement équivalent à celui de l'arabe et où les disciplines scientifiques sont enseignées en français. Ceci est encore valable dans l'enseignement public et dans une 
importante partie de l'enseignement privé, malgré l'installation progressive de l'anglais comme première langue étrangère.

3. Analysant les représentations de l'étranger dans des manuels de langue étrangère, Geneviève ZARATE pose que « la relation entre langue et culture étrangères dépend du contexte national où la langue est enseignée ", et que « la description d'une culture étrangère obéit à des règles particulières lorsqu'elle se situe en contexte scolaire. Ce sont ces règles que nous cherchons à mettre au jour et qui caractérisent ce que nous appellerons désormais la description scolaire ». Celle-ci est " appréhendée comme l'expression d'une entité nationale (...) impliquée dans la construction de l'identité nationale de l'élève » (1993: 11).

4. Précisons que le Liban est le pays le plus représenté dans ces manuels.

5. Bien entendu l'altérité dans les manuels ne se résume pas aux pays francophones, car d'autres pays sont représentés de façon disséminée. Il serait intéressant de les étudier mais pour des raisons de place nous ne les prenons pas en compte dans la présente étude.

6. C'est-à-dire des manuels utilisés dans les établissements où cette langue est privilégiée par rapport à l'anglais, qui n'a alors que le statut de deuxième langue étrangère, et donc un nombre d'heures plus réduit.

7. Du point de vue de l'âge des apprenants, les $4 \mathrm{e}, 5 \mathrm{e}$ et $6 \mathrm{e}$ années du primaire correspondent respectivement au CM1, au CM2 et à la 6e en France. Les 7e, 8e et 9e années du cycle complémentaire correspondent respectivement à la $5 \mathrm{e}$, à la $4 \mathrm{e}$ et à la $3 \mathrm{e}$ du collège français.

8. L'enseignement privé représente au moins la moitié de l'enseignement global. Nous avons exclu les manuels utilisés dans les établissements homologués par l'État français car ce sont des manuels de français langue maternelle faits en France.

9. Dans chacune des deux équipes se trouvait également une co-auteure française.

10. Les citations qui suivent sont tirées du site officiel du CNRDP : http://www.crdp.org/CRDP/ French/curriculum/curriculum.asp

11. À côté de l'enseignement public, l'enseignement privé comprend des établissements laïcs et des établissements religieux reflétant les différentes obédiences confessionnelles. Parmi les dixhuit confessions officiellement reconnues, douze sont chrétiennes (maronites, grecs orthodoxes, grecs catholiques, arméniens orthodoxes, arméniens catholiques, syriaques orthodoxes, syriaques catholiques, coptes, assyriens, chaldéens, catholiques romains, protestants), cinq sont musulmanes (sunnites, chiites, druzes, alaouites, ismaéliens) et une est judaïque. Chaque communauté est autorisée à avoir ses propres écoles pourvu que les objectifs définis par le CNRDP soient suivis.

12. Il arrive même que certains de ces thèmes à vocation citoyenne reviennent d'une année sur l'autre.

13. Livre de français, Éducation de base Septième année, pp.184-190.

14. Ibid., pp.30, 33, 40 et 42-43 respectivement.

15. Dire, Lire, Éducation de base Cinquième année, pp.162-163.

16. Au pays des mots, Éducation de base Quatrième année, pp.182-183 et 189.

17. Au pays des mots, Éducation de base Cinquième année, pp.108-109.

18. Livre de français, Éducation de base Huitième année, pp.106-129.

19. Un grand nombre de textes est d'ailleurs tiré des brochures du Conseil National du Tourisme.

20. Les échanges entre peuples qui sont mentionnés ont lieu de préférence dans l'Antiquité, entre Phéniciens et Égyptiens, et sont de nature commerciale et culturelle.

21. Voir A la croisée des textes, Éducation de base Septième année, pp.50-51, 52-53 et 60-61.

22. Voir Livre de français, Éducation de base Huitième année, p.98. Livre de français, Éducation de base Septième année, pp.196-198. Au pays des mots, Éducation de base 5e année, pp.104-105.

23. Voir Livre de français, Éducation de base Huitième année, pp.142, 146, 152-156 et Sixième année, pp.75-76, 78, 95, et Au pays des mots, Éducation de base Quatrième année, p.82, Cinquième année, p. 121 et Sixième année, p.122. 
24. Voir par exemple Livre de français, Éducation de base Septième année, p.199.

25. Livre de français, Éducation de base Huitième année, pp.36-39 (Unité « Les voyages »).

26. La question de savoir si le français est langue seconde ou étrangère est controversée au Liban, et les réponses apportées divergent selon un clivage chrétien/ musulman, la langue considérée n'ayant pas, pour des raisons historiques et communautaires, le même statut pour les deux communautés. Elle serait ainsi « plus étrangère » pour les musulmans que pour les chrétiens.

27. Il s'agit de responsables de l'enseignement du français dans les établissements scolaires. Dans l'enseignement privé, ils peuvent être chargés de la dotation en manuels de leurs écoles.

28. Si l'on considère les deux langues étrangères privilégiées dans l'enseignement libanais, il apparaît en effet que, traditionnellement, le français est davantage une langue de la communauté chrétienne, tandis que la communauté musulmane est davantage tournée vers l'anglais.

29. Cette expression se trouve dans les textes officiels du CNRDP, et a été employée par plusieurs informateurs.

30. Plusieurs entretiens ont fait apparaître, associées à cet adjectif, les notions de " monde moderne », d'« humanisme », de « droits de l'Homme » ou de « valeurs universelles ».

\section{RÉSUMÉS}

Au Liban, la décennie 1990 a été marquée par la sortie de la guerre et par la mise en œuvre d'une importante réforme éducative mettant la question des valeurs du vivre ensemble et du rapport à l'altérité au centre des préoccupations. Nous abordons ces valeurs et cette éducation à l'altérité telles qu'elles s'énoncent dans les textes officiels d'une part, et telles qu'elles apparaissent dans les manuels de français d'autre part, à travers les représentations du Liban et des pays francophones. La particularité du contexte libanais, entre éducation post-conflit et tensions intercommunautaires encore vives, rend complexes la construction de l'unité nationale et les modalités d'ouverture à l'Autre: la description scolaire revêt ainsi des allures paradoxales, lorsqu'elle entreprend d'éduquer à l'altérité sans représenter la diversité sociale libanaise, ni traiter de la ou des cultures cibles comme de cultures étrangères. Nous décrivons des procédés d'occultation et de contournement divers tout comme nous tentons de cerner le rôle que joue l'enseignement du français dans l'éducation aux valeurs.

In Lebanon, after the 1975-1990 war, an important educational reform emphasized the question of coexistence, respect of otherness and values. In this paper I examine these values and education to otherness as they appear in the official instructions of the ministry of education, and how teaching French textbooks convey them, through representations of Lebanon and of French-speaking countries. Lebanon's particular background, between post-conflict education and strained inter-community relationships, complicates the shaping of national unity and openness to others; the textbooks' discourses become paradoxical when they try to teach a way of living together without showing Lebanese social diversity, nor presenting the target cultures as foreign cultures. I try to highlight how they avoid such taboo subjects, and which role French teaching plays in passing down educational values. 
INDEX

Mots-clés : altérité, français langue seconde (FLS), Liban, manuel scolaire, représentations, valeurs

Keywords : Lebanon, otherness, textbook, values

\section{AUTEUR}

AMANDINE DENIMAL

DIPRALANG, Université Montpellier 3, France 